\title{
The Design of Cutting Force Calculation System for Orthogonal Experiment
}

\author{
LIU Xuebin ${ }^{1,2, a}$, WANG Xibin ${ }^{2, b}$ and Li Chongning ${ }^{1, c}$ \\ ${ }^{1}$ Institute of Mechatronics Engineering, Tianjin University of Technology and Education, Tianjin \\ 300222 \\ ${ }^{2}$ Key Laboratory of Fundamental Science for Advanced Machining, Beijing Institute of Technology, \\ Beijing 100081, China \\ a ewinding@163.com, 'bangxibin@bit.edu.cn, ' Lichongning@tute.edu.cn
}

Keywords: Cutting force, orthogonal experiment, Visual C++.

\begin{abstract}
Based on the design of orthogonal cutting tests, study the processing and the analysis method of orthogonal test data, program the module for solving regression equation, research on the calculation of cutting force curve, drawing and display issues based on the cutting test data which is stored cutting database. The software, developed by using the development plat of Visual $\mathrm{C}++$, can easily deal with orthogonal experiment data , draw and display the fitting equation of the test curve after user enters the relevant parameters according to needs intuitively, has laid the foundation for the draw curve ,such as tool wear, tool life, the chip breaking test. Thus it is enriched the cutting data storage capabilities of the database, and data processing capabilities and test curve processing capabilities.
\end{abstract}

\section{Introduction}

At present, many countries have started to create cutting database which famously is the CUTDATA developed by the United States and the INFOS developed in Germany. Establishment of cutting database can bring considerable economic benefits for the users, cutting database allows processing costs decreased by $10 \%$ or more according to the CIRP survey of the economic benefits of cutting database. Usually the amount of cutting data in cutting database obtained by two ways: cutting testing and cutting manual. Proceed from the design of orthogonal cutting tests, study the calculation of the cutting force curve, drawing and display issues based on the cutting test data stored in the cutting database. Rao[1] research the influence of speed, feed, depth of cut on cutting force and surface roughness by Taguchi method. Patel [2]research the optimization of machining parameters, such as speed, feed, depth of cut, tool geometry, cutting environment, tool material, work material, etc. using the technique of Taguchi and ANOVA. Durai[3] uses the matlab genetic algorithm solver to do the optimization of cutting parameters by experimental design which use the Taguchi method. Badadhe[4] uses the Taguchi orthogonal array method to deal with the response from multi-variables in order to select the combination of optimum cutting parameters which will result in better surface finish. Kilickap[5] select glass fibre reinforced plastic composite as experimental material for investigation of cutting parameters (cutting speed, feed rate and tool geometry) affecting delamination in drilling operation by Taguchi method. Yang[6] uses the Taguchi method, which is a powerful tool to design optimization for quality, to find the optimal cutting parameters for turning operations, employs an orthogonal array, the signal-to-noise $(\mathrm{S} / \mathrm{N})$ ratio, and the analysis of variance (ANOVA) to investigate the cutting characteristics of S45C steel bars using tungsten carbide cutting tools. Çiçek[7] researches the effects of deep cryogenic treatment and drilling parameters on surface roughness and roundness error were investigated in drilling of AISI 316 austenitic stainless steel with M35 HSS twist drills by Taguchi method. Nalbant[8] uses the Taguchi method to find the optimal cutting parameters for surface roughness in turning, employs The orthogonal array, the signal-to-noise ratio, and analysis of variance to study the performance characteristics in turning operations of AISI 1030 steel bars using TiN coated tools. As a powerful tool to design optimization, orthogonal 
experiment or Taguchi method usually is used to research the cutting parameters, a common tool as part of the cutting database is developed to compute the orthogonal experiment data automatically in this paper. This tool contains various utility functions such as fitting the data of experiment, displaying the fitting curve, saving the fitting formula in the database.

\section{Orthogonal Regression Experimental Design}

Cutting force $F_{f}$ usually is affected by three factors(the depth of cut $a_{p}$, feed rate $f$ and cutting speeds $v_{c}$ )in the process of metal cutting. Based on the experimental results, can be calculated in terms of the depth of cut $a_{p}$, feed rate $f$ and cutting speeds $v_{c}$. The empirical formula is as follows $[9,10]:$

$$
F_{f}=C_{F_{f}} a_{p}^{x_{F_{f}}} f^{y_{F_{f}}} v_{c}^{{ }^{z_{F_{f}}}} \text {. }
$$

Where $F_{f}$ is cutting force, $a_{p}$ is the depth of cut, $f$ is feed rate, $v_{c}$ is cutting speeds, the coefficients, $x_{F_{f}}, y_{F_{f}}, z_{F_{f}}, C_{F_{f}}$ are constants obtained from curve fitting.

Logarithmic Eq.(1), then

$$
\ln F_{f}=\ln C_{F_{f}}+x_{F_{f}} \ln a_{p}+y_{F_{f}} \ln f+z_{F_{f}} \ln v_{c} .
$$

Let $y=\ln F_{f}, b_{0}=\ln C_{F_{f}}, b_{1}=x_{F_{f}}, b_{2}=y_{F_{f}}, b_{3}=z_{F_{f}}$, then

$$
y=b_{0}+b_{1} \ln a_{p}+b_{2} \ln f+b_{3} \ln v_{c} .
$$

The Coding of Levels of Factors. In order to scientifically design orthogonal regression test, process the test data using regression analysis, reduce the number of test, solve the regression equation, it is necessary to code the level of factors, establish the correspondence relationship between the level of factors and "coding" by the appropriate conversion to the value of factors, achieve the purpose of simplifying the calculation of the regression equation. Here, given the change interval of the depth of cut $a_{p}$ is $\left[a_{p 1}, a_{p 2}\right]$, the change interval of feed rate $f$ is $\left[f_{1}, f_{2}\right]$, the change interval of cutting speeds $v_{c}$ is $\left[v_{c 1}, v_{c 2}\right]$, the equation is as follows:

$$
\begin{aligned}
& x_{1}=\frac{2\left(\ln a_{p}-\ln a_{p 2}\right)}{\ln a_{p 2}-\ln a_{p 1}}+1 . \\
& x_{2}=\frac{2\left(\ln f-\ln f_{2}\right)}{\ln f_{2}-\ln f_{1}}+1 . \\
& x_{3}=\frac{2\left(\ln v_{c}-\ln v_{c 2}\right)}{\ln v_{c 2}-\ln v_{c 1}}+1 .
\end{aligned}
$$

Thus after coding, the regression problem is transformed into the following:

$$
y=b_{0}+b_{1} x_{1}+b_{2} x_{2}+b_{3} x_{3} .
$$

Determining the Experimental Plan. This experiment has three factors and two levels, using the tables of single(linear) orthogonal regression design, $L_{8}\left(2^{7}\right)$, table1 shows the experimental plan. 
Table1. experimental plan

\begin{tabular}{c|ccccccc}
\hline & $x_{1}$ & $x_{2}$ & $x_{3}$ & $x_{1} x_{2}$ & $x_{1} x_{3}$ & $x_{2} x_{3}$ & $x_{1} x_{2} x_{3}$ \\
\hline 1 & 1 & 1 & 1 & 1 & 1 & 1 & 1 \\
2 & 1 & 1 & -1 & 1 & -1 & -1 & -1 \\
3 & 1 & -1 & 1 & -1 & 1 & -1 & -1 \\
4 & 1 & -1 & -1 & -1 & -1 & 1 & 1 \\
5 & -1 & 1 & 1 & -1 & -1 & 1 & -1 \\
6 & -1 & 1 & -1 & -1 & 1 & -1 & 1 \\
7 & -1 & -1 & 1 & 1 & -1 & -1 & 1 \\
8 & -1 & -1 & -1 & 1 & 1 & 1 & -1 \\
\hline
\end{tabular}

Calculate Regression Coefficient. According to the experimental design eight tests will be need, if the test results are $y_{i}(i=1,2, \mathrm{~L}, 8)$, then a regression mathematical model is as follows:

$$
y_{i}=\beta_{0}+\beta_{1} x_{i 1}+\beta_{2} x_{i 2}+\beta_{3} x_{i 3}+\varepsilon_{i}(i=1,2, \mathrm{~L}, 8) .
$$

The structure matrix is

$$
X=\left[\begin{array}{lrrr}
1 & x_{11} & x_{12} & x_{13} \\
1 & x_{21} & x_{22} & x_{23} \\
\mathrm{M} & \mathrm{M} & \mathrm{M} & \mathrm{M} \\
1 & x_{81} & x_{82} & x_{83}
\end{array}\right] .
$$

Obtain the least squares estimation of the parameters $\beta$

$$
b_{j}=\frac{1}{8} \sum_{i=1}^{8} x_{i j} y_{i}(j=0,1,2,3) \text {. }
$$

Thus

$$
\hat{y}=b_{0}+b_{1} x_{1}+b_{2} x_{2}+b_{3} x_{3} \text {. }
$$

Significance Test. After otained regression equation, it is necessary to conduct the statistical tests, which can test the significant of the regression equation and coefficients.

Significance test of regression equations usually use F-Test, given the significance level , statistics can be calculated by the following equation.

$$
F=\frac{\frac{4}{3} \times 8 \sum_{j=1}^{3} b_{j}{ }^{2}}{\sum_{i=1}^{8} y_{i}{ }^{2}-\frac{1}{8}\left(\sum_{i=1}^{8} y_{i}\right)^{2}-8 \sum_{j=1}^{3} b_{j}{ }^{2}}>F_{\alpha}(3,4) .
$$

So, regression equation significant in the significant level $\alpha$.

While significance test of regression coefficient use F-Test too, given the significance level $\alpha$, statistics can be calculated by the following equation.

$$
F_{j}=\frac{4 \times 8 b_{j}{ }^{2}}{\sum_{i=1}^{8} y_{i}{ }^{2}-\frac{1}{8}\left(\sum_{i=1}^{8} y_{i}\right)^{2}-8 \sum_{j=1}^{3} b_{j}{ }^{2}}>F_{\alpha}(1,4) \quad(j=0,1,2,3) \text {. }
$$

So, regression coefficient significant in the significant level $\alpha$. 
Solve the Regression Equation. After substitution variable by Eq.(4)-Eq.(6), the regression equation is gain.

$$
\begin{aligned}
y=b_{0} & +b_{1} x_{1}+b_{2} x_{2}+b_{3} x_{3} \\
=b_{0} & +b_{1}\left(\frac{2\left(\ln a_{p}-\ln a_{p 2}\right)}{\ln a_{p 2}-\ln a_{p 1}}+1\right) \\
& +b_{2}\left(\frac{2\left(\ln f-\ln f_{2}\right)}{\ln f_{2}-\ln f_{1}}+1\right)+b_{3}\left(\frac{2\left(\ln v_{c}-\ln v_{c 2}\right)}{\ln v_{c 2}-\ln v_{c 1}}+1\right)
\end{aligned}
$$

\section{Software System Design}

Using access database to store test data, VC6.0 as a development tool, the software system is developed on the development platform VC6.0, mainly to calculate the coefficient of the regression equation and draw fitting curve, based on the algorithm of drawing curve[11,12]. System diagram shown in Fig.1.

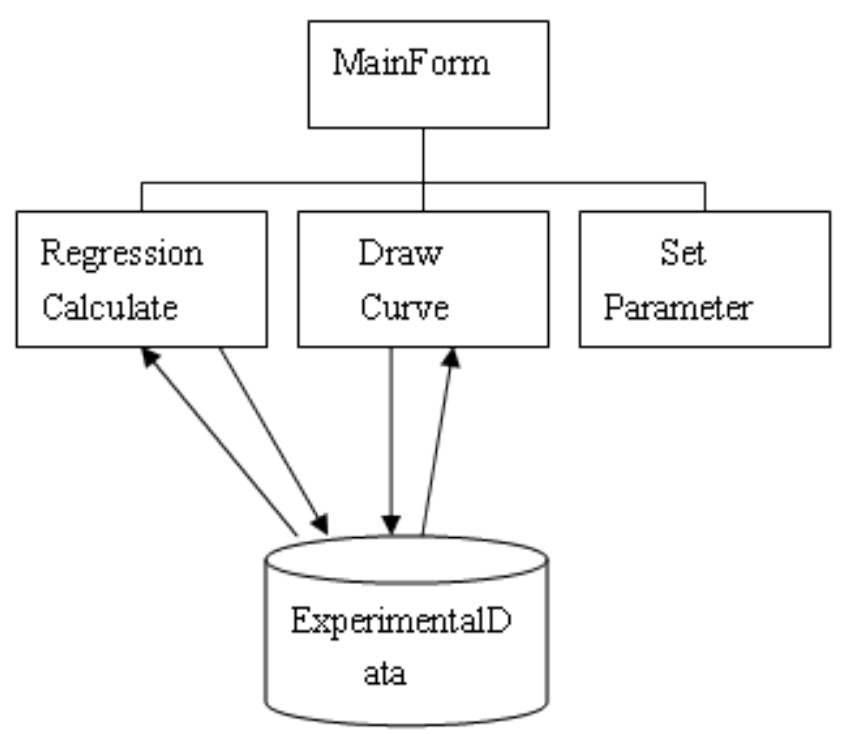

Fig.1 The system structure

The run result of the software shown in Fig.2-Fig.5.

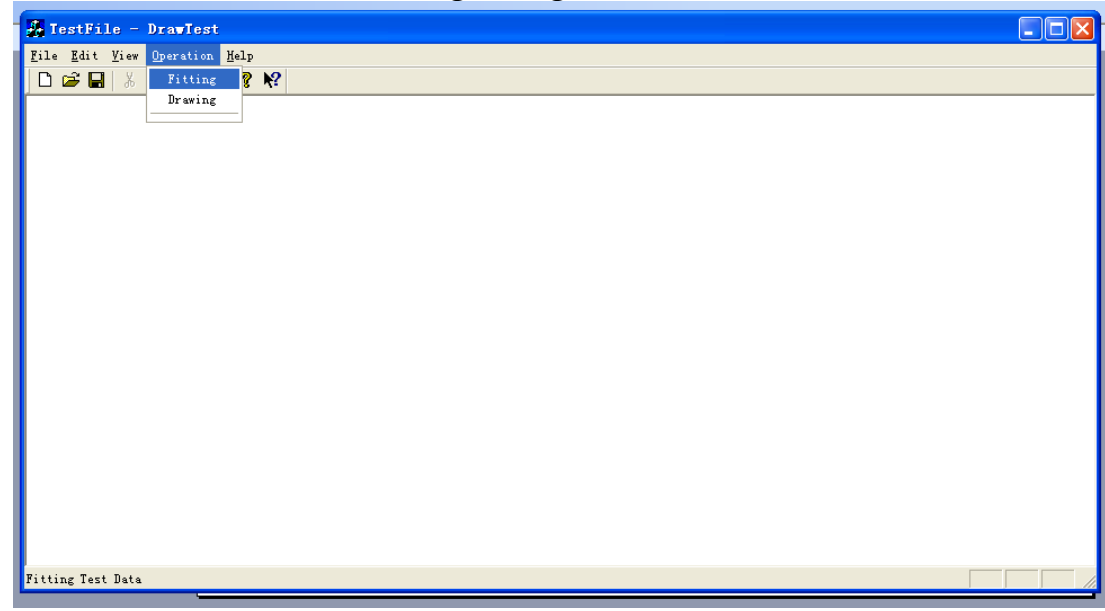

Fig.2 The interface of the software 


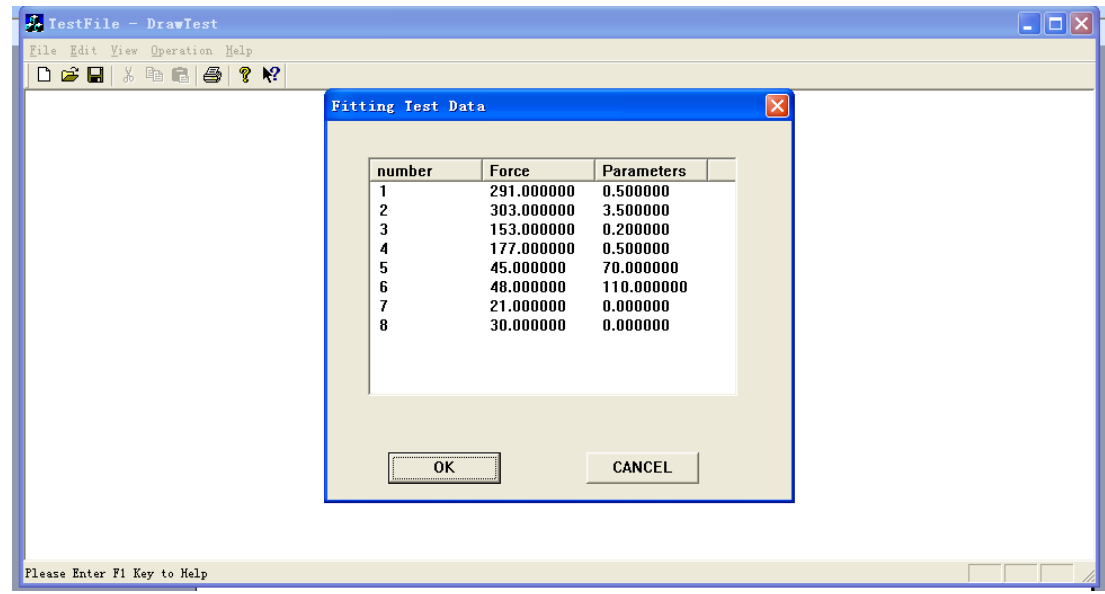

Fig.3 The experimental Data

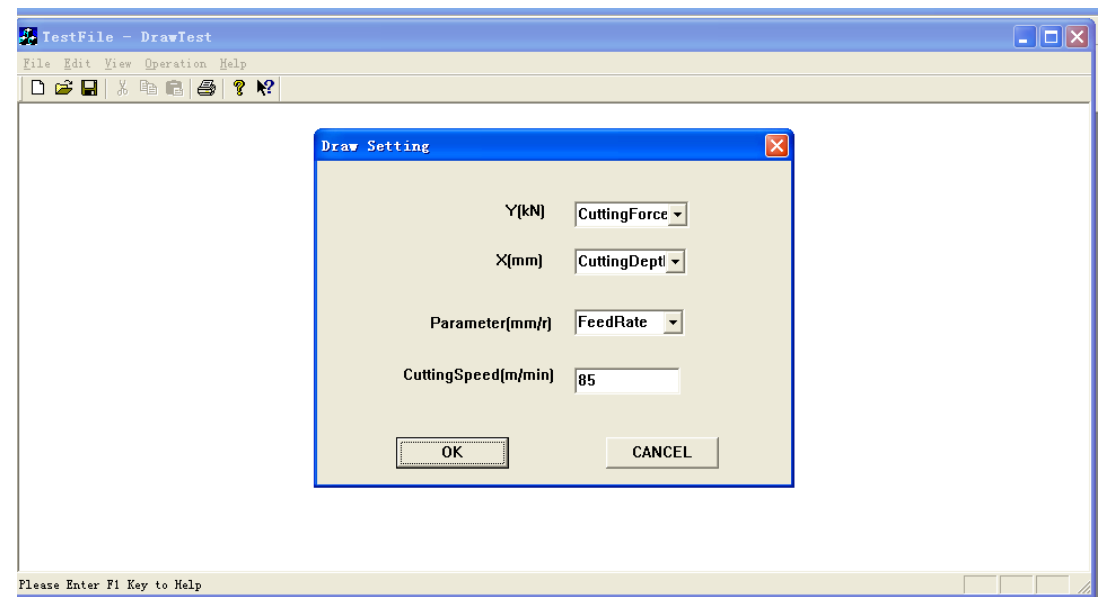

Fig4 Setting parameter

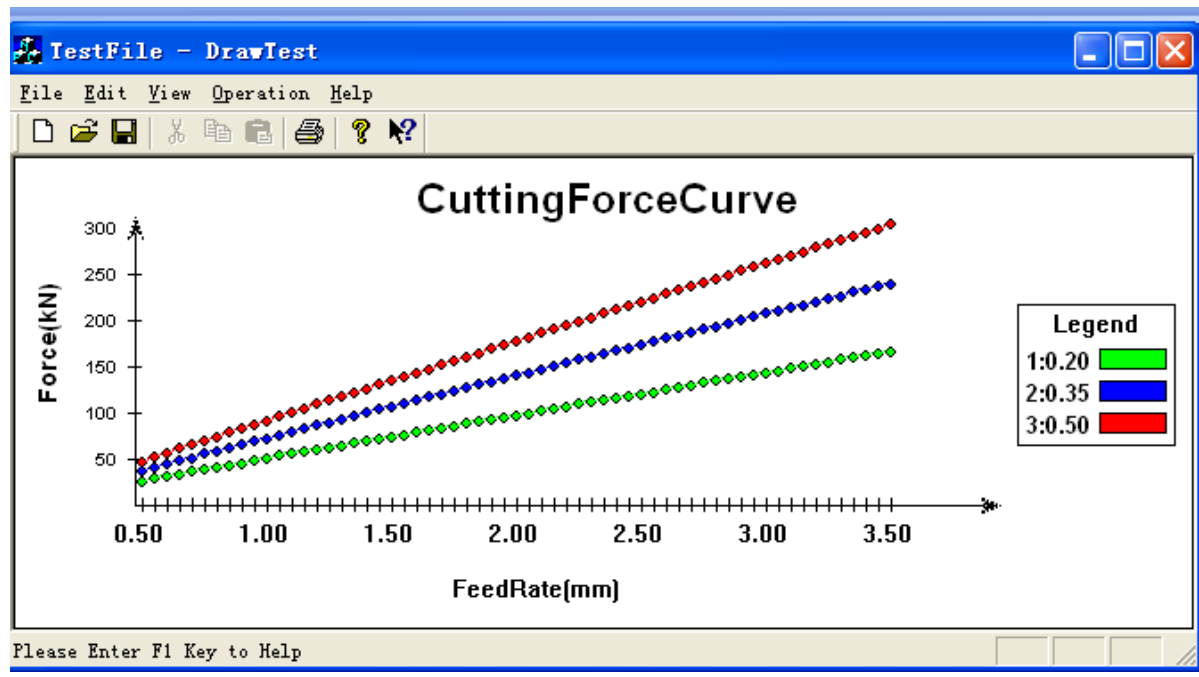

Fig.5 Draw curve

\section{Conclusions}

With the program designed and developed in this paper , regression orthogonal experimental data can be easily calculated and can carry out the drawing and display of the fitted curve, making the results more intuitive, lay the foundation for drawing tool wear curve , tool life curve, chip breaking test curve. Thus it is enriched the cutting data storage capabilities of the database, and data processing capabilities and test curve processing capabilities. 


\section{References}

[1] C.J. Rao, D. Nageswara Rao, P. Srihari: Influence of Cutting Parameters on Cutting Force and Surface Finish in Turning Operation. Procedia Engineering, Vol.64(2013),p.1405-1415

[2] Mihir T. Patel, Vivek A. Deshpande: Optimization of Machining Parameters for Turning Different Alloy Steel Using CNC -Review. International Journal of Innovative Research in Science, Engineering and Technology,Vol.3(2014),p.9423-9430

[3] Durai Matinsuresh BABU, Mouleeswaran Senthil KUMAR, othiprakash VISHNUU: optimization of cutting parameters for cnc turned parts using TAGUCHI's technique. International Journal of Engineering, Vol.3(2012),p.493-496

[4] A.M.Badadhe, S. Y. Bhave, L. G. Navale: Optimization of cutting parameters in boring operation. IOSR Journal of Mechanical and Civil Engineering (IOSR-JMCE), Vol.6(2013),p. 10-15

[5] Erol Kilickap: Determination of optimum parameters on delamination in drilling of GFRP composites by Taguchi method. Indian Journal of Engineering \& Materials Sciences, Vol.17(2010),p. 265-274

[6] W.H. Yang, Y.S. Tarng: Design optimization of cutting parameters for turning operations based on the Taguchi method.Journal of Materials Processing Technology, Vol. 84(1998), p.122-129

[7] Adem Çiçek, Turgay Kıvak, Gürcan Samtaş: Application of Taguchi Method for Surface Roughness and Roundness Error in Drilling of AISI 316 Stainless Steel. Journal of Mechanical Engineering, Vol.58(2012),p. 165-174

[8] M. Nalbant, H. Gökkaya, G. Sur: Application of Taguchi method in the optimization of cutting parameters for surface roughness in turning. Materials and Design , Vol.28(2007), p.1379-1385

[9] LU Jian-hong, SUN Jia-ning: Metal cutting tool (4th) (China Machine Press , China 2005)

[10] YANG He,HAN Mei: Modeling method of materials processing experiments (Northwestern Polytechnical University Press, China 2008)

[11]LIU Xue-bin: Development of the Testing Bad Data Software for Large-Diameter Measuring Apparatus. MODULAR MACHINE TOOL \& AUTOMATIC MANUFACTURING TECHNIQUE, Vol.6(2006),p.3-5

[12] LIU Xue-bin, GUO Li-bin, LAN Ying-ying: Establishment of Mathematical Model by Means of New On-line Method of Measurement of Part Dimension of Large Diameter and Numerical Analysis of Its Mathematical Model. APPLIED SCIENCE AND TECHNOLOGY, Vol.30(2003),p.1-3 